\title{
WAVE REFLECTION GENERATED BY CAISSONS WITH INTERNAL RUBBLE MOUND OF VARIABLE SLOPE
}

\author{
Carla Faraci ${ }^{1}$, Biagio Cammaroto ${ }^{2}$, Luca Cavallaro ${ }^{3}$ and Enrico Foti ${ }^{4}$
}

\begin{abstract}
The paper reports on an experimental campaign focused on the performances of prefabricated caissons with internal rubble mound (combined caissons) in terms of incident wave energy reduction. The frontal opening and the chamber width of the caisson were changed in order to find the optimal design dimensions allowing the reflection coefficient to be reduced as much as possible. As expected, the best performances of the combined caisson occur when the rubble mound slope is gentler. The experimental results were also compared with an analytical model available in literature; such a comparison showed that the combined caisson exhibits a smaller reflection than predicted as far as wave periods higher than 8-9 s at a prototype scale are concerned.
\end{abstract}

Keywords: rubble mound; combined caissons; reflection coefficient.

\section{INTRODUCTION}

The development of global trade and ship transportation often requires that the existing docks must be upgraded, consolidated or enlarged, in order to face effectively the increasing demand of people and freight traffic.

To this aim, quay over piles having an absorbing slope below the structure, can be used to enlarge or restructure existing quays, particularly in presence of foundation soils having good geotechnical characteristics.

As regards the wave energy dissipation within the harbor basin, the absorbing slope behaves generally better but the overall structure requires a lot of horizontal space, which is not always available, due to construction problems or to other specific limitations; in many cases perforated caissons, even though characterized by higher reflection coefficients, are often preferred as they do not require too much space.

Experiments on the main factors influencing the wave energy dissipation of perforated caissons have been performed since the heuristic work of Jarlan (1961) and Marks and Jarlan (1968). Laboratory experiments have been conducted by Suh et al (2001) for the reflection of irregular waves of various significant wave heights and periods impinging upon breakwaters having various wave chamber widths. $\mathrm{Li}$ et al. (2003) investigated the reflection of obliquely incident waves by breakwaters with a doublelayered perforated wall. Brossard et al. (2003) performed experiments in a wave flume to analyse the interaction between regular gravity waves and a fixed absorbing semi-immersed breakwater, investigating effects of both the immersion and the width of the structure.

Many efforts have been put in the analytical investigation of the wave energy reflection as well. Based upon linearized shallow water wave theory, Kondo (1979) developed an analytical model for calculating reflection coefficient of a perforated-wall caisson breakwater having one or two perforated walls. He found that the breakwater having two perforated walls could give much lower reflection coefficient than that having one perforated wall. Fugazza and Natale (1992) and Bennett et al. (1992) have also theoretically analyzed the reflection coefficients of a perforated caisson and the wave forces on it by using the potential flow theory. In particular, Fugazza and Natale showed that the reflection is minimized when the wave chamber width is about one quarter of the wave length. In contrast to the conclusion made by Kondo (1979), Fugazza and Natale showed that the perforated-wall breakwater with a single wave chamber could provide the largest reduction of wave reflection in the range of practical applications. Suh and Park (1995) developed an analytical model that can predict the reflection coefficient of a perforated wall caisson mounted on a rubble mound foundation when waves are obliquely incident to the breakwater at an arbitrary angle. The model was then extended by Suh et al. (2001) to predict the reflection characteristics of irregular waves incident on perforated structures through a frequency-average method, and by Suh et al (2006) in order to consider partially perforated wall caissons. Takahashi et al. (2002) reported on the reflection performances of perforated wall caissons by means of VOF numerical techniques. Chen and Li et al. (2004) calculated the reflection

\footnotetext{
${ }^{1}$ Department of Civil Engineering, University of Messina, C.da di Dio, S. Agata,98166, Messina ITALY

2 Department of Civil Engineering, University of Messina, C.da di Dio, S. Agata,98166, Messina ITALY

${ }^{3}$ Department of Civil Engineering, University of Messina, C.da di Dio, S. Agata,98166, Messina ITALY

${ }^{4}$ Department of Civil and Environmental Engineering, University of Catania, v.le A. Doria, 6, 95125, Catania, ITALY
} 
coefficient for irregular waves on perforated caissons and the total horizontal force on those caissons through the equivalent energy-component method. Liu et al (2007) examined the reflection of obliquely incident waves by an infinite array of partially perforated caissons, on the basis of the linear potential theory.

Caisson-type porous seawalls wave been also thoroughly studied. Madsen (1983) proposed a theoretical solution for the reflection of linear shallow-water waves from a vertical homogeneous rubble-mound structure with impermeable core. Postma (1989) performed random wave flume tests on rock slopes investigating the effects of permeability, slope angle, wave height and length on wave reflection, providing also empirical formulations for the reflection coefficient determination.

Zhu and Chwang (2002) set up a physical model simulating Victoria harbor in Hong Kong. They showed that caisson seawall structures with a suitable porosity can dissipate a remarkable portion of the incident wave energy. They also observed that for relatively short waves, there is little difference in the reflection behavior between a fully immersed structure and a half immersed one.

A combination of these two kinds of wave absorbing breakwaters, namely the perforated and the rubble mound caisson, consists of a concrete caisson with large gaps and a porous slope inside. Indeed such a caisson does not require too much horizontal space and, due to its internal rubble mound, should perform well in wave reflection control. This kind of caisson, hereinafter referred to as combined caisson, boasts some applications for harbor enlargements. For example, the Port of Siracusa (Italy), originally built by the ancient Greeks, in order to increase the touristic traffic, was interested by a growth plan. The harbor is located in a very large and shallow natural embayment, with a narrow inlet where, due to the extension and the orientation of the basin, the waves generated by the wind within the bay can be substantial and may affect the operations within the port. Moreover, the harbor basin is extremely important from an archaeological point of view, thus the caissons to be used not only have to be suitable for reducing wave reflection, but also their size has to be limited, due to the constraints imposed in order to preserve the archaeological findings onto the sea bottom. In this case the designer proposed the adoption of combined caissons as the presence of the rubble mound slope within the caisson cell dissipates the energy of the incident wave motion, thus reducing the reflected wave and, at the same time, the width of the caisson itself is not too large, so that the occupation of soil is minimized as much as possible. More recently, a similar application was also proposed for the site of Augusta (Italy), a commercial harbor where the adaptation of an existing quay in order to support heavy loads was designed through combined caissons broken up by piles.

However, in spite of their breaking through importance, an exhaustive literature about such caissons and particularly about its reflective characteristics is not yet available. In order to contribute to fill such a gap the present paper reports the results of an experimental campaign aimed at investigating the performances of the combined caisson in terms of reflection coefficient when its design dimensions change. The paper is organized as follows: first the non dimensional analysis of the problem is reported, then the experimental set up is described. The subsequent section presents the laboratory measurements of wave reflection, afterward the experiments and the main results are discussed; the paper ends with some conclusions.

\section{DIMENSIONAL ANALYSIS}

The parameters that have been considered to the aim of a dimensional analysis of the problem, according to Hudson (1979) are the following:

$\mathrm{d}$ water depth at the toe of the structure;

$\mathrm{H}$ wave height;

L wavelength (or wave period T);

$\mathrm{U}_{0}$ wave orbital velocity;

g gravitational acceleration;

$\mu$ dynamic viscosity of water;

$\rho$ water density;

$\rho_{\mathrm{a}}$ density of armor units;

$\mathrm{l}_{\mathrm{a}}$ characteristic linear dimension of armor unit;

$\xi_{\mathrm{a}}$ characteristic linear dimension of armor unit surface roughness;

$\alpha$ rubble mound slope angle measured from the horizontal (or caisson frontal opening $\mathrm{h}$ );

b chamber width. 
In Figure 1 a sketch of the adjustable caisson along with the previously mentioned geometrical parameters is reported.

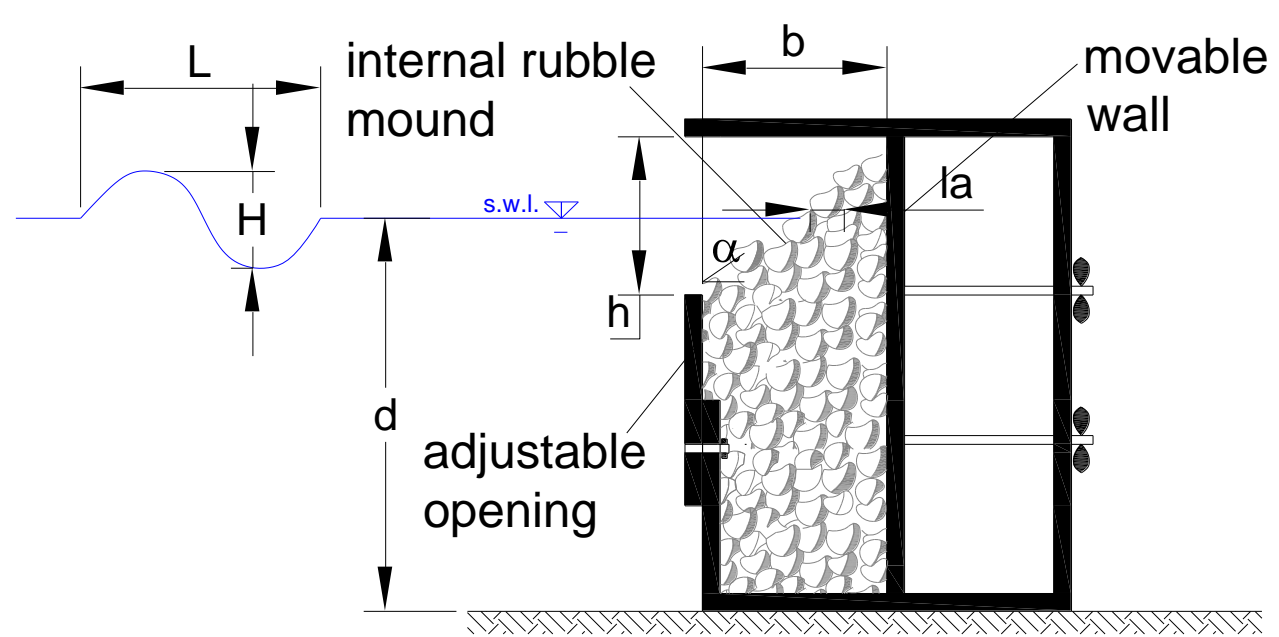

Figure 1. Sketch of the adjustable caisson with the involved geometrical quantities.

Since the main aim of the experimental investigation is to focus on wave reflection induced by the caisson rather than on the stability of the armor layer, the damage to rubble mound has not been taken into account among the aforementioned parameters.

A function will exist linking the previous quantities, such that:

$$
f\left(d, H, L, U_{0}, g, \mu, \rho, \rho_{a}, l_{a}, \xi_{a}, \alpha, b\right)=0
$$

These can be arranged in terms of non dimensional products as:

$$
f\left(\frac{d}{L}, \frac{H}{L}, \frac{U_{0}}{\sqrt{g d}}, \frac{\rho U_{0} d}{\mu}, \frac{\rho}{\rho_{a}-\rho}, \frac{l_{a}}{d}, \frac{\xi_{a}}{l_{a}}, \alpha, \frac{b}{L}\right)=0
$$

Some of the previous non dimensional parameters do not change during the experimental campaign, namely:

$$
\frac{\rho}{\rho_{a}-\rho}, \frac{l_{a}}{d}, \frac{\xi_{a}}{l_{a}}
$$

Moreover Reynolds number relevance is marginal if compared to the other non dimensional variables, as viscous effects are contained within bottom boundary layer, and the flow through the armor layer is turbulent.

Thus equation (2) can be simplified as:

$$
f\left(\frac{d}{L}, \frac{H}{L}, \frac{U_{0}}{\sqrt{g d}}, \alpha, \frac{b}{L}\right)=0
$$

The model has been scaled according to a Froude criterion assuming a geometrical scale ratio equal to $1: 40$.

\section{EXPERIMENTAL SET-UP}

The experiments have been carried out at the Hydraulics Laboratory of the University of Messina. The experimental apparatus is made up by a wave flume $18.5 \mathrm{~m}$ long, with a cross section of $0.4 \times 0.8$ $\mathrm{m}$. It has a inox steel flat bed and glass walls, made by panels of $1.25 \times 0.8 \mathrm{~m}$. The flume has a tilting bed and an external recirculating system feeded by a $11 \mathrm{~kW}$ centrifugal pump, and it can be suitably 
used also for steady flow tests. It is held by two supports: the first, located approximately $3 \mathrm{~m}$ far from one end of the flume, is an hydraulic piston that allows the slope of the channel to be set, the other support, $10 \mathrm{~m}$ far from the first, is a hinge fulcrum. In the present paper it has been kept in an horizontal configuration, and a third support has been added for safety reasons, at a $3.5 \mathrm{~m}$ distance from the second one.

In Figure 2a an ensemble view of the wave flume is reported; in Figure $2 b$ a sketch of the set up is also reported.

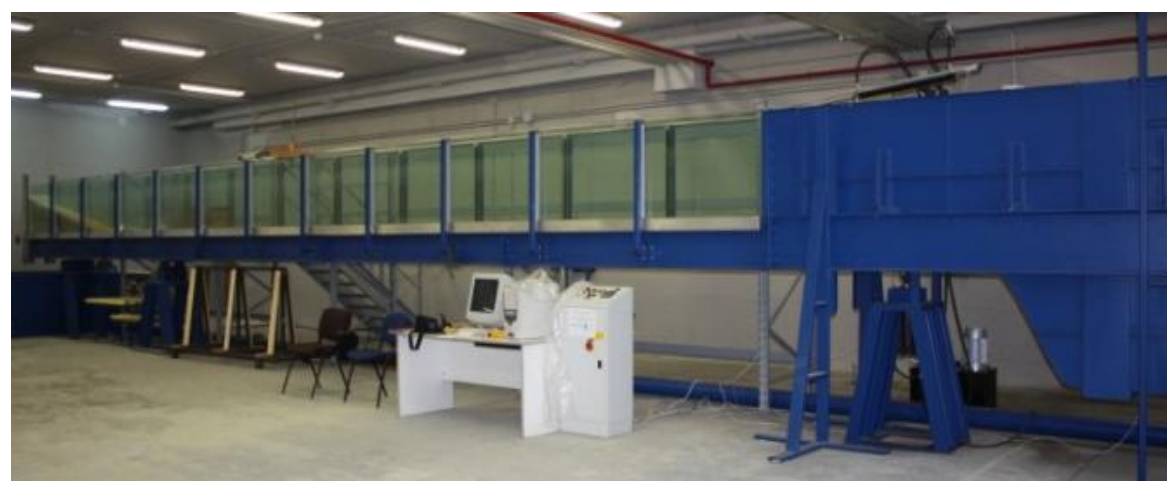

(a)

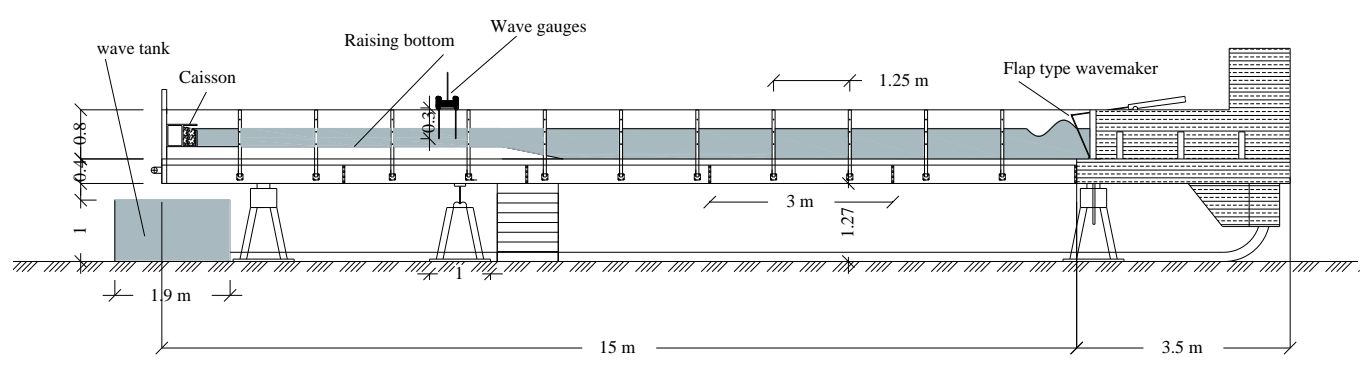

(b)

Figure 2. (a): Ensemble view of the wave flume; (b) Sketch of the wave flume.

A flap type wavemaker, mounted at one end of the channel, has been used to generate waves within the flume. The wave maker is driven by a pneumatic system and electronically controlled. Both regular and random waves can be reproduced. Regular waves are controlled by a control panel that allows frequency, amplitude and offset position of the signal to be set, while random waves are generated through a software which is able to reproduce a Jonswap spectrum. In this latter case a time series of 5 minutes is generated and it is identically repeated for all the experiment duration. At the back of the wavemaker some mattresses of creased pipe pieces have been located with the aim to absorb any spurious reflection caused by the flap.

At the opposite side of the flume a PVC bulkhead closes the channel and just in front of it a scaled model of an adjustable combined caisson, at 1:40 geometrical scale ratio, has been placed. In Figure 3 a picture of the adjustable combined caisson is reported. The caisson is made of steel and is composed by a double chamber in the wave crest direction and a vertical bulkhead in the frontal part, that can slide in order to permit the regulation of the caisson frontal opening. The chamber width can be varied by adjusting several screws at the back of the caisson. The inner part of the caisson has been filled with rubble mound, in order to simulate a rock armour. The armour element has been modeled by means of marble pebbles whose mean dimension was about $2-3 \mathrm{~cm}$. 


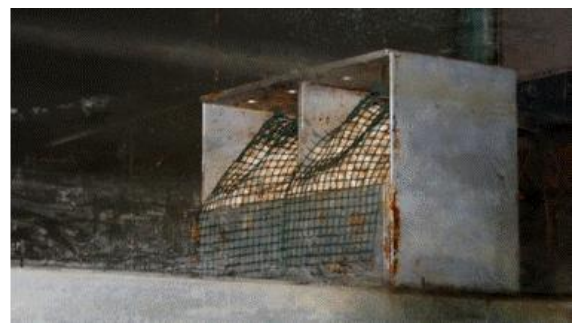

Figure 3. Picture of the adjustable caisson that was adopted in the present experimental campaign.

Since the main aim of the work was to analyze the wave reflection induced by the caisson when both the chamber width and the frontal opening were changed, several resistive level gauges have been located along the flume. As it will be better described in the following section, two wave gauges have been placed approximately one wavelength offshore the caisson in order to measure the reflection coefficients due to the presence of the caisson itself, while other two gauges have been located respectively 6 and $9 \mathrm{~m}$ far from the wavemaker, in order to control the wave height along the flume.

\section{LABORATORY MEASUREMENT OF WAVE REFLECTION}

Several methods for estimating wave reflection in laboratory facilities have been presented in literature, in the presence of both regular waves (Isaacson, 1991) and irregular waves (Thornton and Calhoun, 1972; Goda and Suzuki, 1976; Mansard and Funke, 1980).

The method of Goda and Suzuki (1976) is based on the estimation of the incident wave spectra and includes the presence of two level gauges separated by a small distance parallel to the wave direction. This method starts from the assumption that a system of multi reflected wave trains is present in a channel. The waves propagate toward the structure through a superposition of the original incident waves. The technique is based on the assumption that the wave height observed in channel is equal to the square root of the sum of the squares of the incident and reflected waves height.

The application presupposed that the gauge closer to the reflecting surface should be positioned at least at one wavelength from it to obtain the spectra. Goda and Suzuki (1976) developed the method using the Fourier transform, which as became a standard in laboratory measurements of wave reflection.

The method developed by Mansard and Funke (1980) is based on the least squares principle that allows the noise in the signal for all the three probes to be minimized. This method focuses on phase differences between them and allows a better stability in the estimations and a greater range of frequency resolution, with less dependence on the distance between the probes. Laboratory tests of Mansard and Funke (1980) showed a good agreement between the measurements of the spectra of the incident waves calculated by the least squares method and the corresponding spectra measured simultaneously in an adjacent channel without a reflective structure.

As the method of Goda and Suzuki(1976), it is based on the linear theory and assumes that the probes are placed over a flat bottom. The main advantage of this method is to make the wave reflection analysis less sensitive to the noise contaminating the measurements (Isaacson 1991). As with previous approach the Mansard and Funke (1980) method exhibits singularities if the three probes are equidistant; the method breaks down when the spacing between two adjacent probes in an integer number of half wave lengths as for the previous method.

In the present work, however, the method proposed by Goda and Suzuki (1976) has been adopted. Indeed an initial comparison between Goda and Suzuki (1976) and Mansard and Funke (1980) approaches has been attempted for few experiments and, since no noticeable differences were observed, the two probes method has been selected. With reference to the scheme reported in Figure 4, the amplitude of the incident and the reflected waves, $a_{i}$ and $a_{r}$ respectively, are measured by means of two wave gauges located in front of the reflective wall, spaced by the distance $\Delta$. The interested reader may refer to the original paper for the analytical details. However, it is worth pointing out here that the method breaks up for wavelengths which do not follow in the range $0.05<\Delta L<0.45$. 


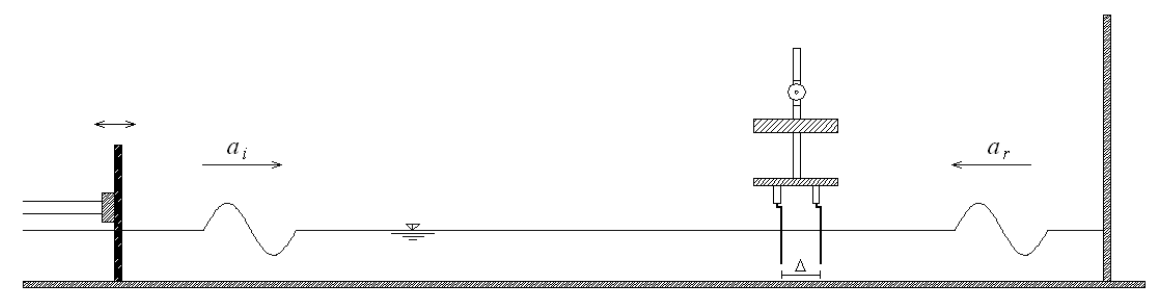

Figure 4 Schematization of the measurement of the incident and the reflected waves in a laboratory flume by means of two wave gauges located in front of the reflective wall and spaced by the distance $\Delta$, as prescribed by the method of Goda and Suzuki, 1976.

\section{EXPERIMENTS}

The experiments were aimed at obtaining the reflection coefficients and the energy spectra in several configurations of the caisson. The signal has been acquired from two level gauges placed at a distance $\Delta$ equal to $45 \mathrm{~cm}$ one from each other.

Physical modeling has been carried out by using a geometrically undistorted scale equal to 1:40 with respect to the prototype, and by guaranteeing Froude and Strouhal similarities. Since the Reynolds number is not the same in the model and in the prototype, reflected waves tend to be higher in the models than in the prototype. Indeed viscous effects in the model can be important, since the structure does not behaves like a porous one. Such a laboratory scale effect has been overcome by increasing the dimension of the stones, whose $\mathrm{D}_{50}$ was set equal to $2.5 \mathrm{~cm}$, with respect to the geometrical scale.

Measurements of the reflection coefficients for a number of wave conditions for the combined caisson with a rubble mound slope have been performed, by changing six different frontal openings (h ranging between 0.07 and $0.12 \mathrm{~m}$ ) and seven different chamber widths (b ranging between 0.08 and $0.18 \mathrm{~m}$ ). By changing the geometrical characteristics and the physical parameters 946 tests were performed.

Regular waves have been characterized by wave heights between 0.01 and $0.08 \mathrm{~m}$ and by wave periods between 0.5 and $2 \mathrm{~s}$. The water depth was kept constant during all the experiments and equal to $\mathrm{h}=0.225 \mathrm{~m}$. It is worth pointing out that during the test runs, in order to avoid any movement of the rubble mound when exposed to waves, a plastic net has been fixed over the pebbles.

A brief summary of the test condition ranges is provided in Table 1.

\begin{tabular}{|c|c|c|c|c|}
\hline & Model sce & & Prototype & \\
\hline & minimum & maximum & minimum & maximum \\
\hline $\mathrm{H}[\mathrm{m}]$ & 0.01 & 0.08 & 0.4 & 3.2 \\
\hline $\mathrm{T}[\mathrm{s}]$ & 0.5 & 2 & 3.2 & 12.6 \\
\hline $\mathrm{D}[\mathrm{m}]$ & 0.225 & 0.225 & 9 & 9 \\
\hline $\mathrm{h}[\mathrm{m}]$ & 0.07 & 0.12 & 2.8 & 4.8 \\
\hline $\mathrm{b}[\mathrm{m}]$ & 0.08 & 0.18 & 3.2 & 7.2 \\
\hline
\end{tabular}

As regards the calculation of the reflection coefficient, once collected from the wave gauges, the surface elevation data are post-processed in order to obtain the measured energy spectra, by using a Direct Fourier Transform (DFT) analysis. Then, the spectra of the incident and reflected waves are calculated by applying the Goda and Suzuki (1976) method. The knowledge of such energy spectra allows for an estimate of both the incident and the reflected wave height, and then in turn of the reflection coefficient $K_{r}$.

In Figure 5 an example of the water elevation recorded at the two wave gauges that have been used for reflection measurements is shown. Similarly, in Figure 6 is reported an example of incident and reflected wave spectra. 


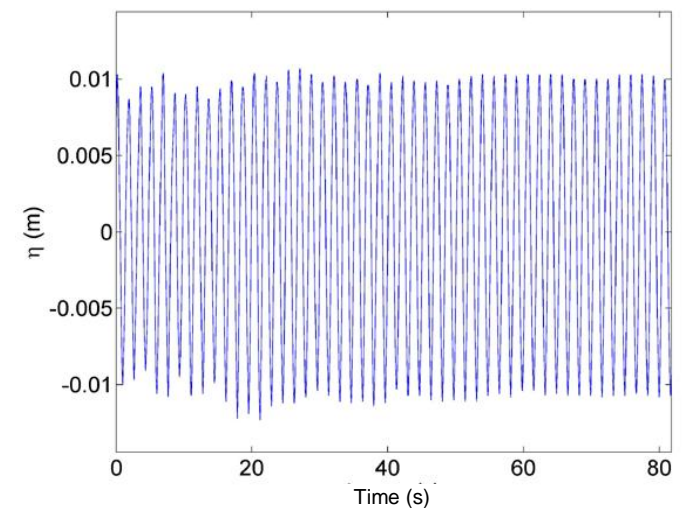

(a)

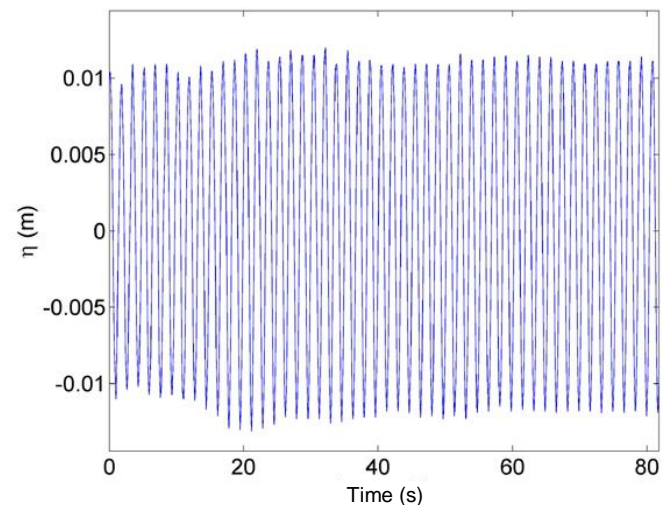

(b)

Figure 5. Water elevation as recorded at the two gauges used for reflection measurements (a): gauge 1 (offshore) (b): gauge 2 (onshore).

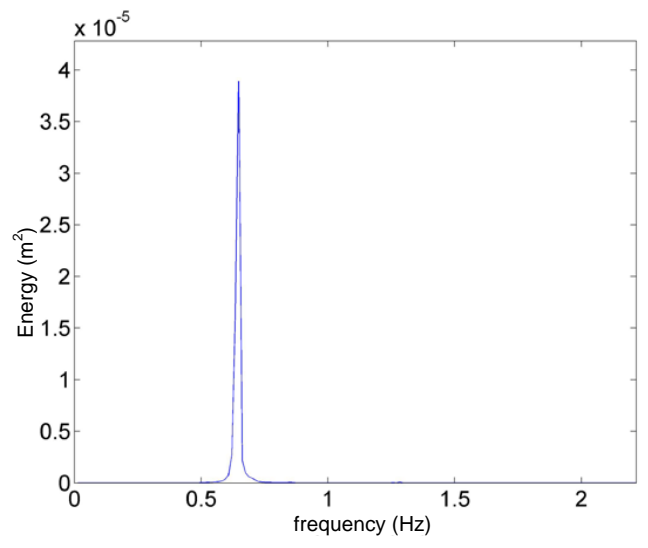

(a)

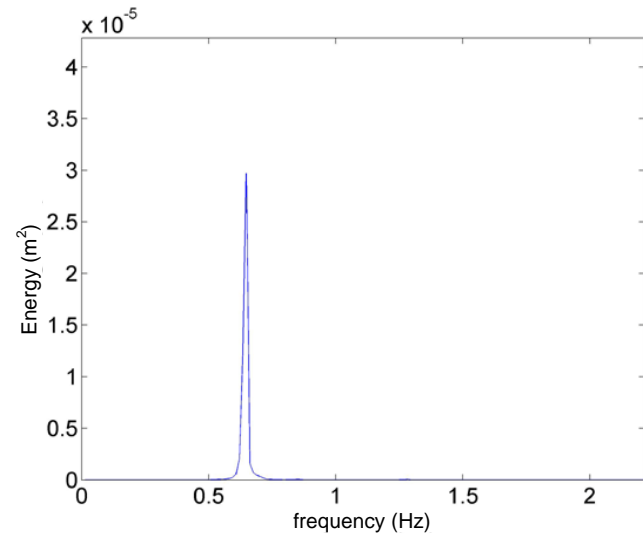

(b)

Figure 6. (a): Incident and (b): reflected wave spectra.

\section{EXPERIMENTAL RESULTS AND DISCUSSION}

The reflection coefficients have been estimated for all the investigated configurations, and the results have been arranged in terms of different chamber widths $b$ and frontal opening $h$, corresponding to various rubble mound slope $\alpha$.

An example is shown in Figure 7, where the reflection coefficients obtained for different frontal opening $\mathrm{h}$ and keeping constant the chamber widths are plotted versus the wave period. A general trend is that the reflection coefficient increases as the period increases. However, when the frontal opening is changed there is not a net behavior of $k_{r}$, probably because the benefits of reducing the rubble mound slope are partly counterbalanced by the augment of totally reflecting surface.

In Figure 8 the same coefficients are arranged for constant frontal openings and by changing the chamber width. In this case, as expected, there is a strong correlation of $k_{r}$ with the chamber width $b$ : indeed since $h$ is constant, the rubble mound slope decreases and wave reflection is dumped as much as $b$ increases. 


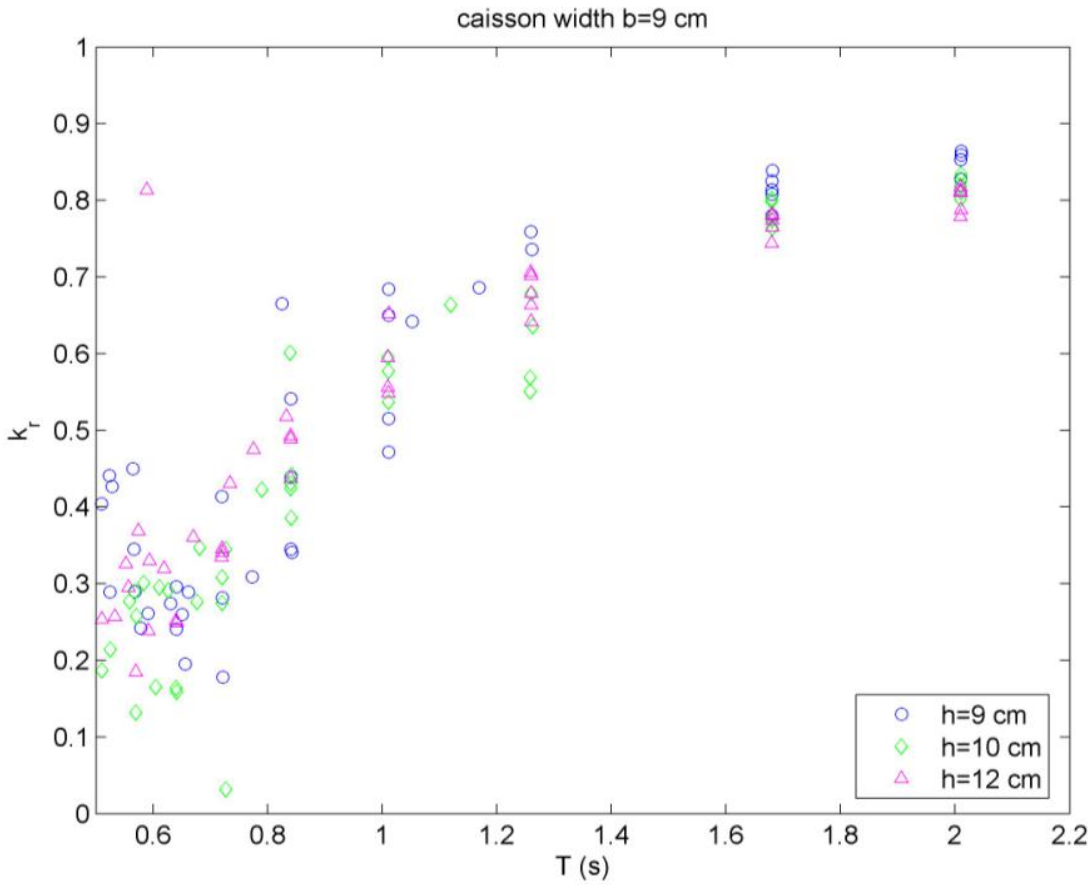

(a)

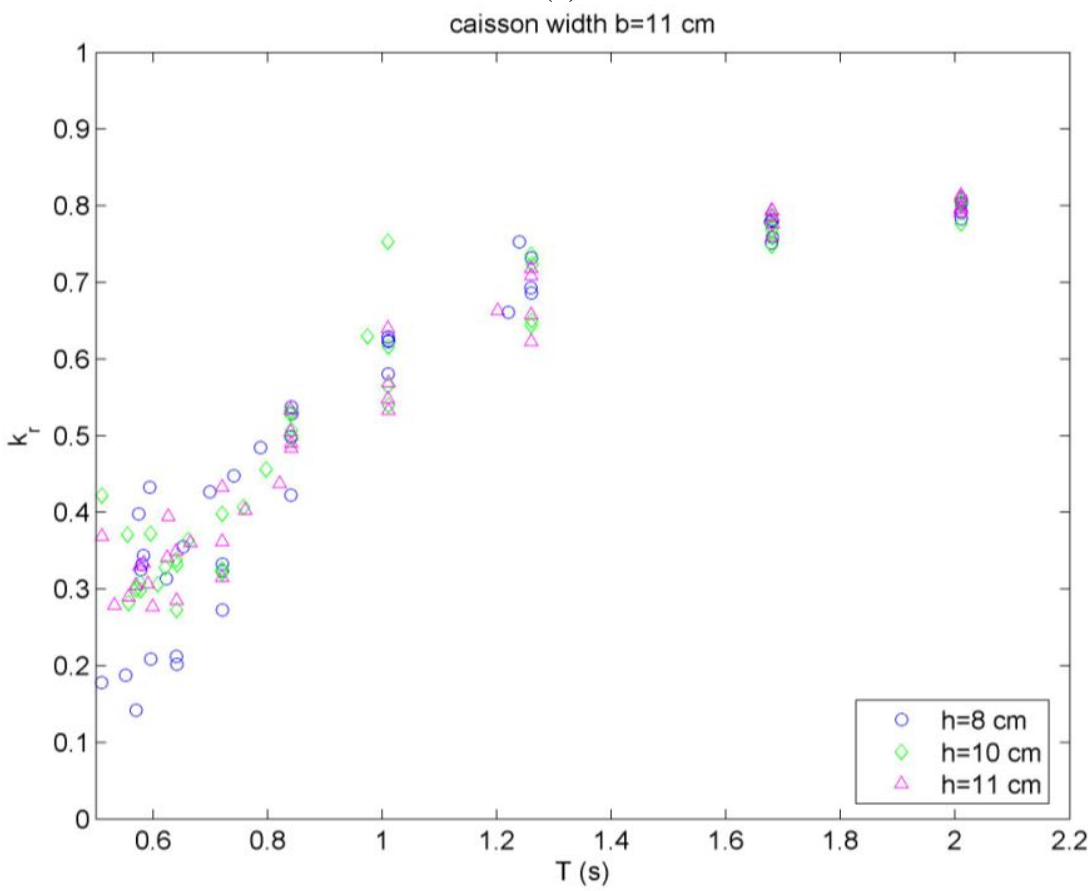

(b)

Figure 7. Reflection coefficients obtained by keeping fixed the chamber width $b$ and varying the frontal opening $h$ : (a) chamber width $b=9 \mathrm{~cm}$; (b) chamber width $b=11 \mathrm{~cm}$;. 


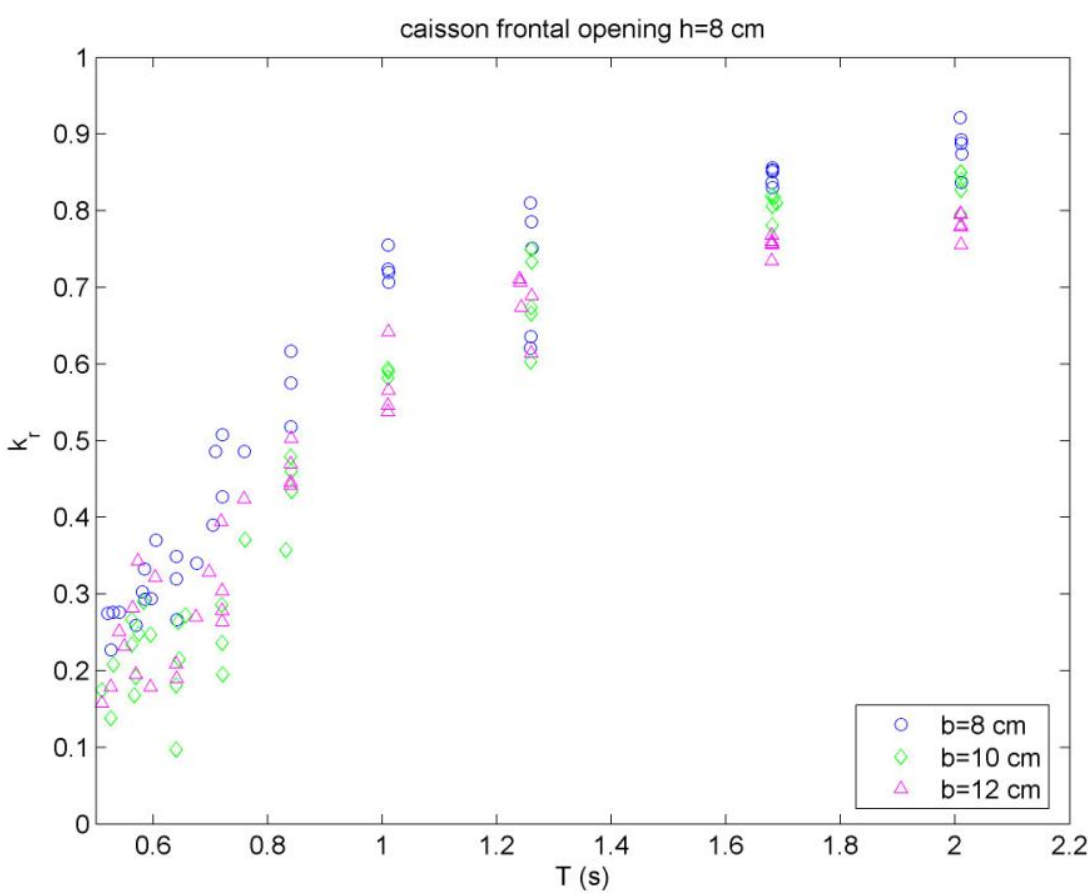

(a)

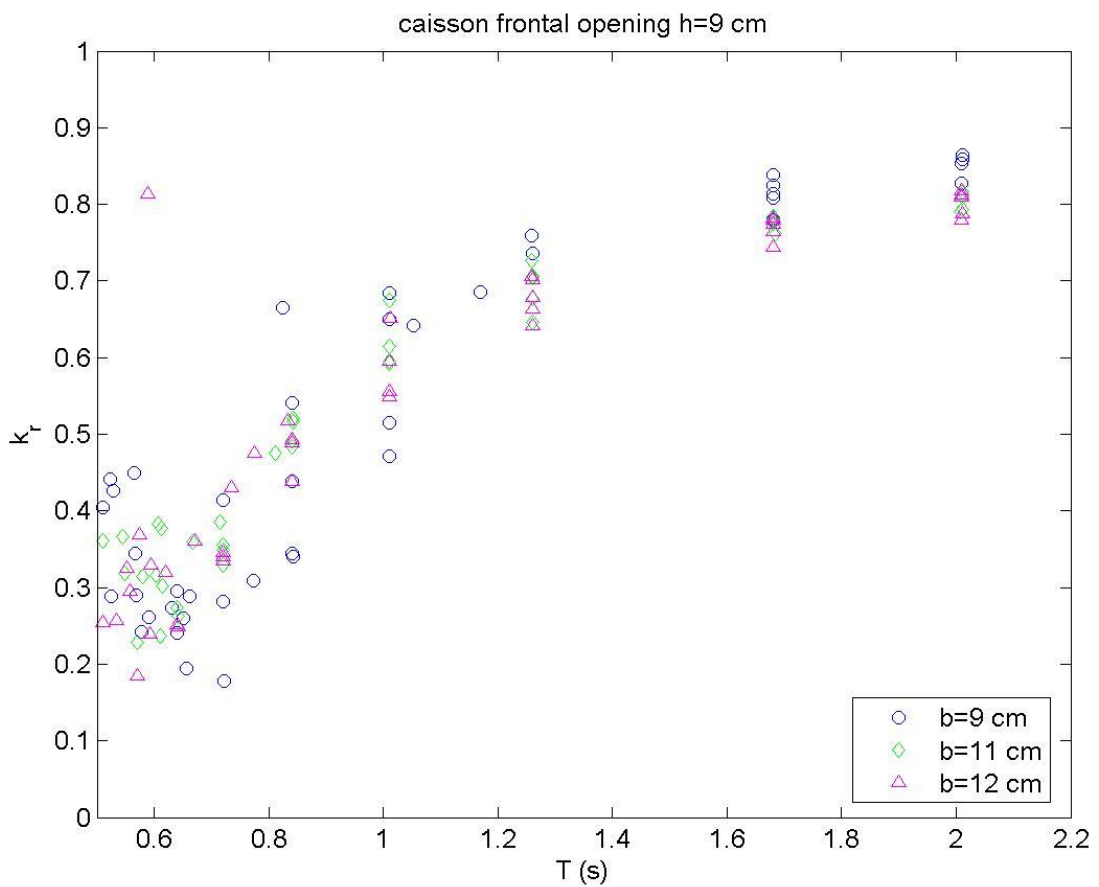

(b)

Figure 8. Reflection coefficients obtained by keeping fixed the frontal opening $h$ and varying the chamber width $b$ : (a) frontal opening $h=8 \mathrm{~cm}$; (b) frontal opening $h=9 \mathrm{~cm}$.

Starting from this result it was possible to recover an empirical relation that allows the reflection coefficient to be estimated as a function of chamber width $b$ and the minimum chamber width $b_{\text {min }}$, i.e. the width corresponding to a 1:1 rubble mound slope $\alpha$. Such relation is:

$$
k_{r}=(1-b)-1.9 \cdot \exp \left\{-2\left[1-4\left(b-b_{\min }\right)\right] \cdot T+\left(b-b_{\min }\right)\right\}
$$


and it is plotted in Figure 9 for the same data showed in Figure 8(a).

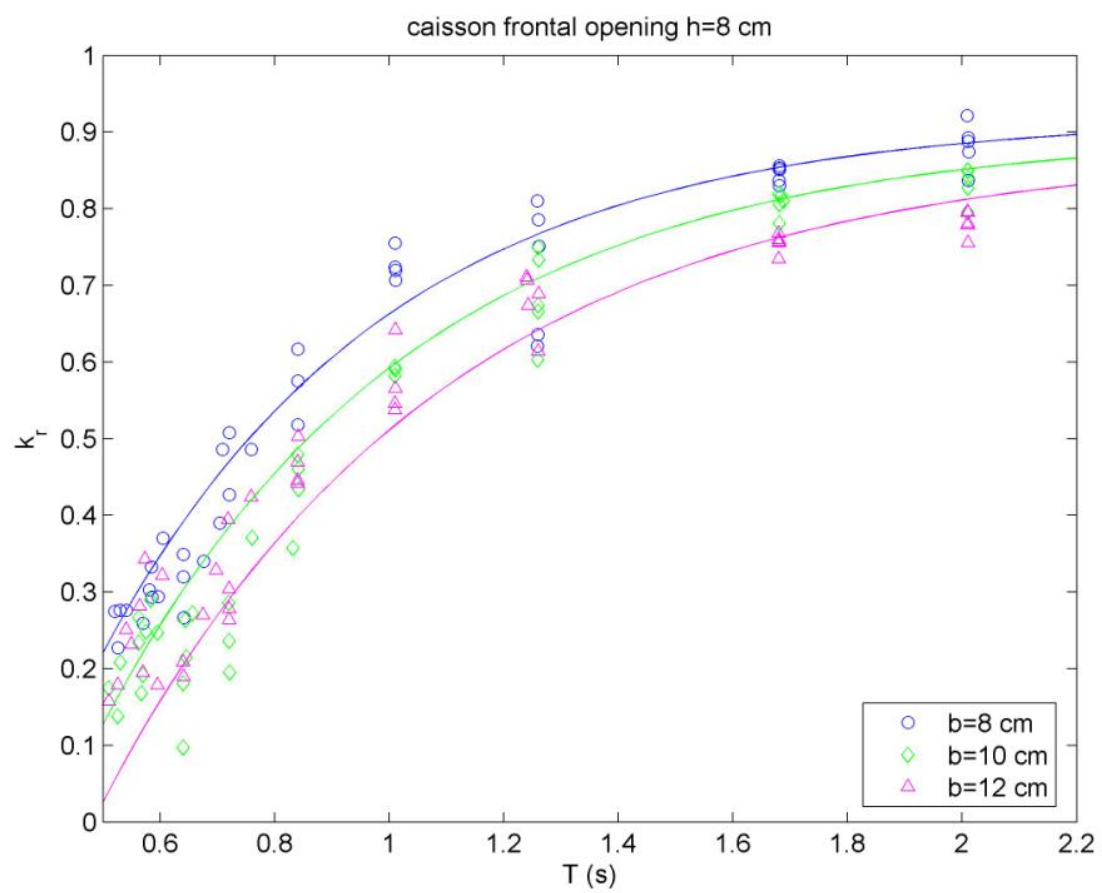

Figure 9.Adaptation of the proposed empirical relation to the experimental data.

As observed in the previous plots, the reflection coefficient is mainly governed by the wave period (or, equivalently, by the wavelength). However, according to the dimensional analysis, among the wave dependent quantities, also the $d / L$ and $H / L$ parameters should be taken into account. It is worth pointing out that the water depth was not changed during the experimental campaign, thus the parameter that is worthy to be considered is just the relative wave height $H / L$.

Already Suh et al (2006) found that increasing wave steepness leads to a reduction in the reflection coefficient, owing to an increase in the energy dissipation within the breakwater at higher wave steepnesses. Also in the present case a similar effect takes place: indeed as the wave height increases it is possible to observe a reduction of the reflection coefficient. This is shown in Figure 10, where among the whole data set, the coefficients obtained for two different wave heights, namely $H=0.02 \mathrm{~m}$ and $H=0.05 \mathrm{~m}$, and for the same frontal opening $h$, i.e. $0.1 \mathrm{~m}$, have been selected.

Two considerations can be done here. The first relates to the differences in the geometry of Suh et al (2006) caisson and the investigated combined caisson. Indeed Suh et al (2006) caisson presents in the frontal wall a set of vertical slots. Notwithstanding the different configuration, the $H=0.05 \mathrm{~m}$ reflection coefficients show a good agreement with the values obtained by Suh et al (2006). The second concern regards the typical shape of the $k_{r}$ versus $b / L$ plot. According to by Fugazza and Natale (1992), this shows a minimum at $b / L$ equal to 0.25 , where partial standing wave is formed due to the wave reflection from the breakwater presents its node. Hence the largest energy loss would occur at this distance. Suh et al (2006) showed that due to the inertia resistance at the perforated wall, a phase difference occurs between inside and outside of the wave chamber in such a way that the perforated wall slows the waves. Consequently the location of the node will move onshore, and the distance where the largest energy loss is gained becomes smaller than L/4. Therefore, the minimum reflection occurs at a value of $b / L$ smaller than 0.25 . In the present case a minimum is visible at $b / L$ equal to about 0.18 only for the smaller wave heights; however it is rather a relative minimum than an absolute one. This result can be partly associated to the presence of a mild slope that allows for greater energy dissipation than a steep one, such effect being more pronounced for smaller wave periods or higher values of $b / L$. 


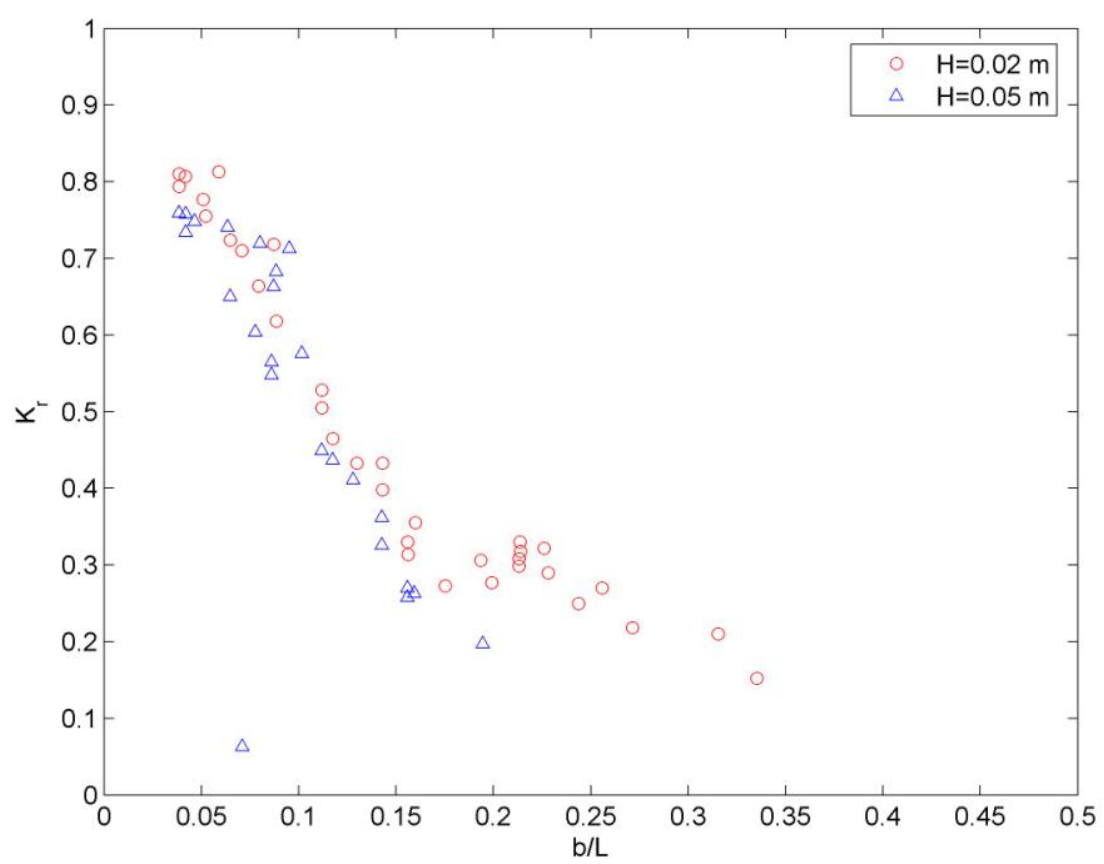

Figure 10. Variation of reflection coefficients with $b / L$ for different values of wave heights.

A comparison with the design formula proposed by Fugazza and Natale (1992) for the minimum reflection coefficient in perforated breakwaters has been reported in Figure 11. More precisely, a comparison between measured and calculated $k_{r}$ is here shown. Also in this case the same observation that has been already done for Suh et al (2006) comparison, concerning the differences in the caisson geometry must be taken in mind.

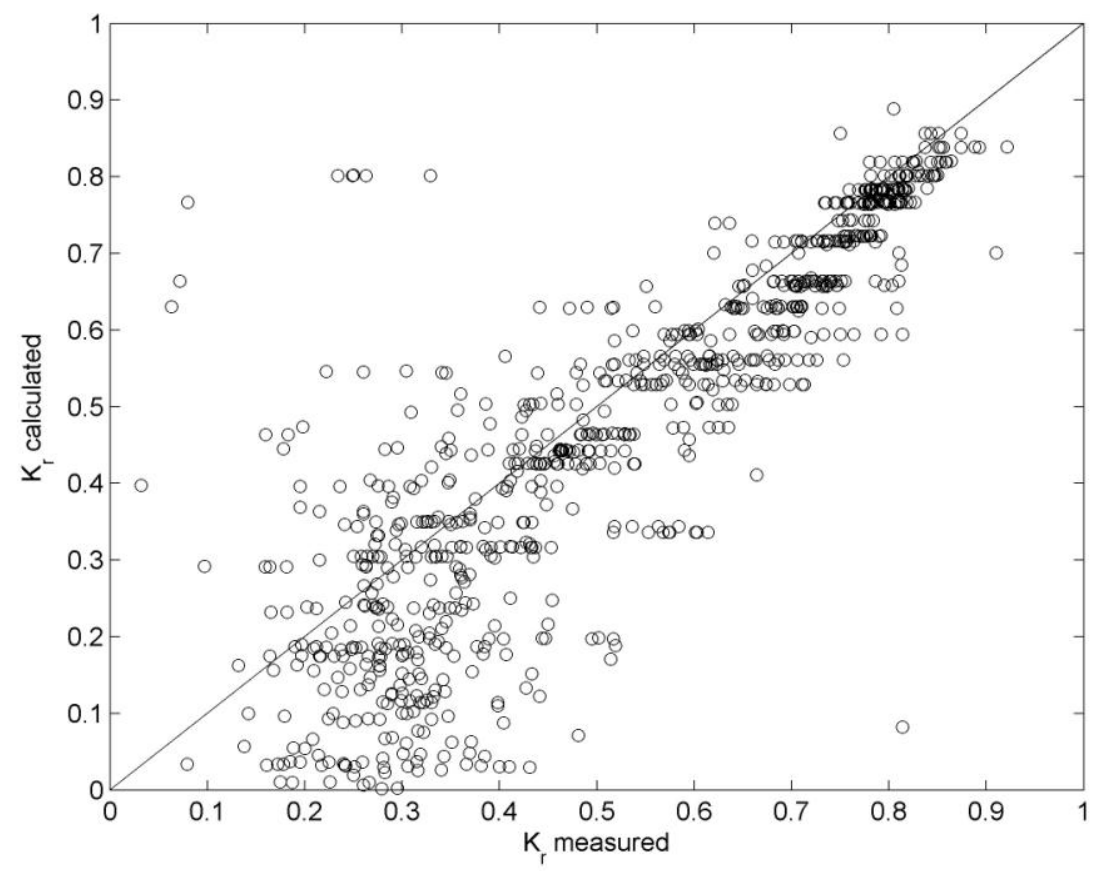

Figure 11. Comparison between measured reflection coefficients and calculated ones through minimum design formula by Fugazza and Natale (1992). 
Here the higher values of reflection coefficients show a good agreement with the design formula proposed by Fugazza and Natale (1992); this occurs for low values of $b / L$, i.e. for greater periods. In this case the performances of the caisson with internal rubble mound is fairly similar to that of the perforated breakwater. For large $b / L$, when the reflection coefficients are smaller, the agreement is missing, due to large dispersion of points with respect to the bisector; however generally the measured values are much higher than the calculated ones. The same data have been thus compared with the analytical formulation proposed by the same authors adapted for the case being. The result is shown in Figure 12, where again measured versus calculated reflection coefficients are plotted.

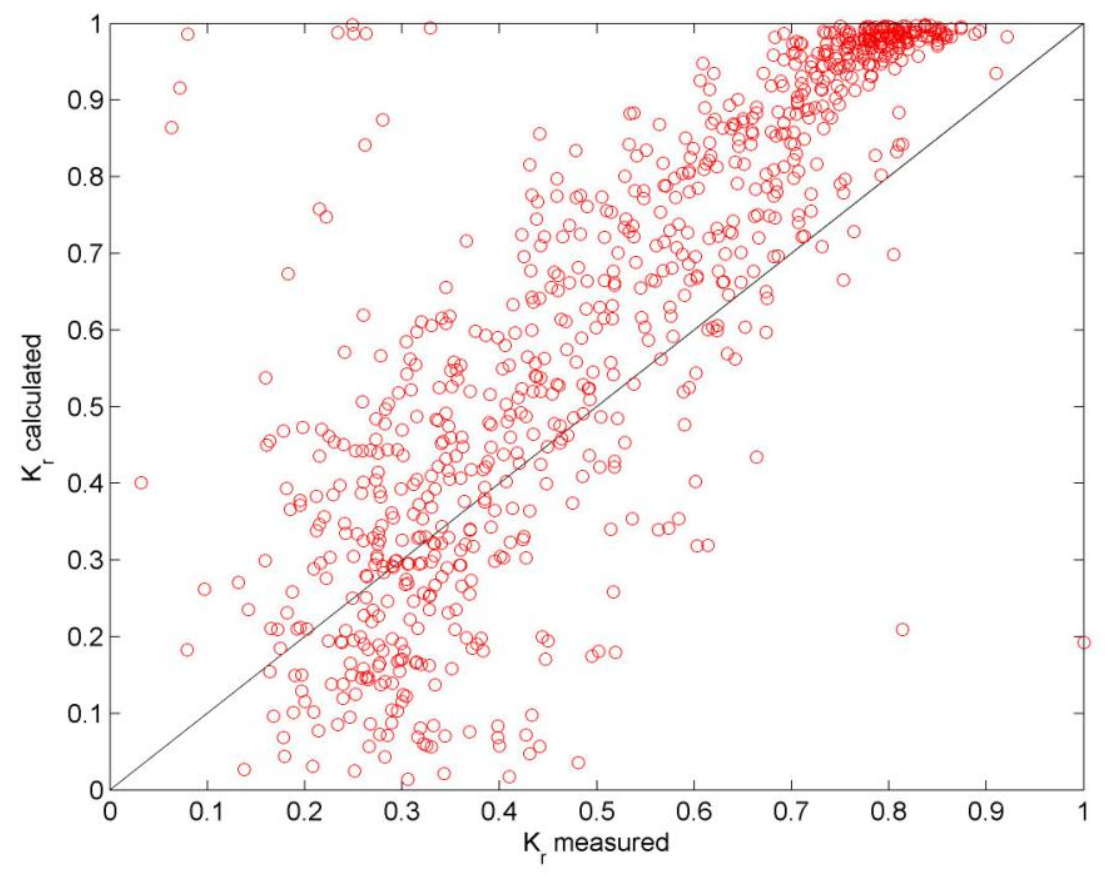

Figure 12. Comparison between measured reflection coefficients and calculated ones through Fugazza and Natale (1992) approach.

It is clearly visible from Figure 12 that, partly due to the differences in the caisson geometry, there is again a large dispersion of points with respect to the bisector. However, for higher wavelengths, i.e. large values of $k_{r}$, the measured value is significantly smaller than the calculated one. A better adaptation can be found after some playing with the geometrical parameters introduced by Fugazza and Natale (1992). In particular the following quantities have been modified with respect to their initial shape:

Jet length

$$
L_{j e t}=\frac{b^{2}}{h} \frac{H}{d}
$$

Wall porosity

$$
r=C_{c} \frac{h^{2}}{d b}
$$

Contraction coefficient

$$
C_{c}=0.6+2.5 \frac{H}{L}
$$

In these relations the presence of a porous rubble mound with a slope $\alpha=b / h$ has been introduced. The result is shown in Figure 13, where a better adaptation of the calculated values to the measured ones can be observed. 


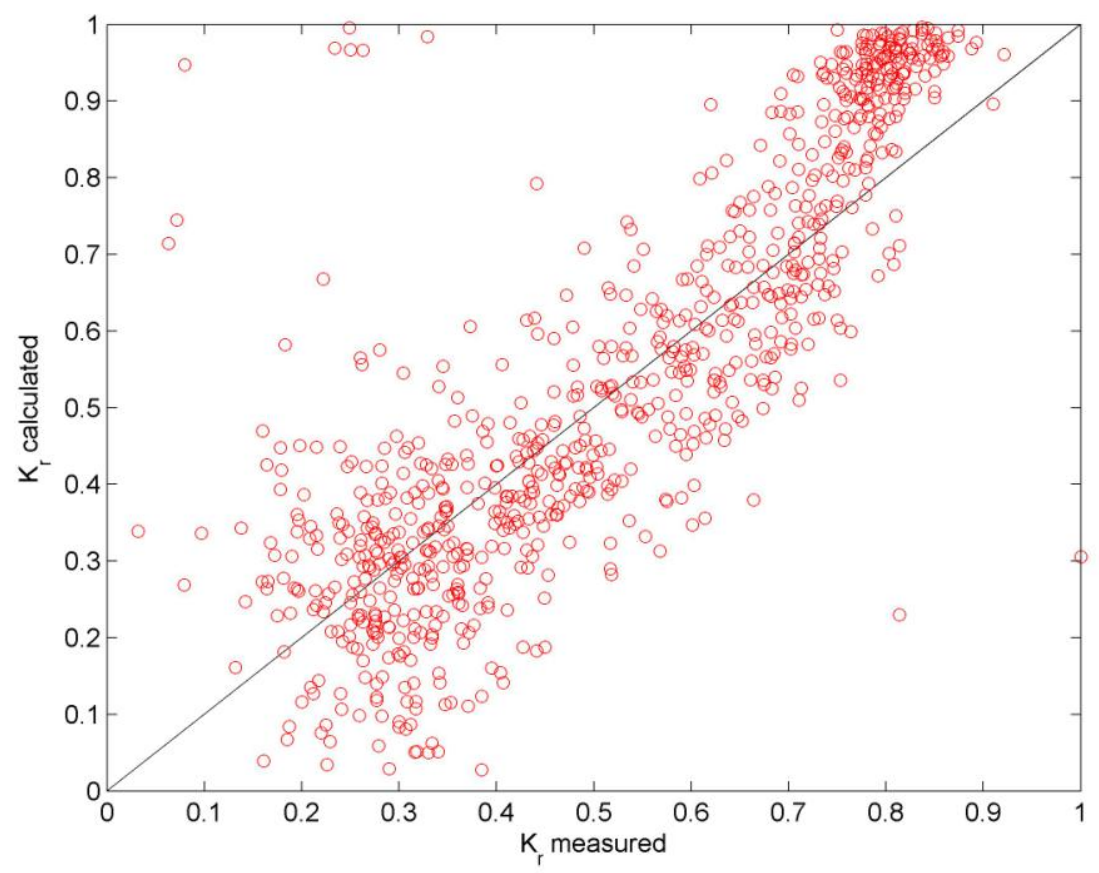

Figure 13. Comparison between measured reflection coefficients and calculated ones through modified Fugazza and Natale (1992) approach.

\section{CONCLUSIONS}

An experimental campaign has been here carried out focusing on the measurement of reflection coefficients in front of a combined caisson with internal rubble mound. The physical model was built with a 1:40 Froude criterion scaling. In the lab the caisson was realized, in order that both the chamber width and the frontal opening could be changed, with the aim to suggest the optimal dimensions of these geometrical parameters to minimize wave reflection within harbor basins. Wave heights and periods were varied in order to reproduce conditions that at a prototype scale could be representative of natural conditions.

Goda and Suzuki (1976) method was applied in the present investigation to calculate wave reflection. The reflection coefficients have been estimated for all the investigated configurations, in terms of different chamber widths $b$ and frontal opening $h$, corresponding to various rubble mound slope $\alpha$.

It was found that the reflection coefficient increases as the period increases. However, when the frontal opening is changed there is not a net behavior of $k_{r}$, probably because the benefits of reducing the rubble mound slope are partly counterbalanced by the augment of totally reflecting surface. Besides, keeping constant the frontal opening, when the rubble mound slope decreases, wave reflection is dumped as much as $b$ increases. An empirical relation that allows the reflection coefficient to be provided as a function of chamber width $b$ and the minimum chamber width $b_{\text {min }}$, has been estimated.

As already observed by Suh et al (2006), as the wave height increases it is possible to observe a second order effect in the reduction of the reflection coefficient.

The experimental results were also compared with Fugazza and Natale (1992) analytical model, showing that the combined caisson exhibits a smaller reflection than literature results as far as wave periods higher than 8-9 s at a prototype scale are concerned. 


\section{ACKNOWLEDGMENTS}

This work has been partly funded by the Italian Minister of University and Research (PRIN 2008 "Strumenti operativi per la stima della vulnerabilita dei litorali sabbiosi anche in presenza di strutture costiere").

\section{REFERENCES}

Bennett, G.S., McIver, P., Smallerman, J.V., 1992. A mathematical model of a slotted wavescreen breakwater. Coast. Eng. 18, 231-249.

Brossard, J. Jarno-Druaux, A., Marin, F., Tabel-Aoul, E.H., 2003. Fixed absorbing semi-immersed breakwater. Coastal Engineering, 49: 25-41.

Chen, X.F., Li, Y.C., 2005. Study of regular wave force on components of perforated caissons with top cover. China Offshore Platform 20 (1), 1-10.

Fugazza, M., Natale,L., 1992. Hydraulic performance of perforated breakwater. J. Waterway, Port,

Coastal and Ocean Eng., 118: 1-14.

Goda, Y., Suzuki, Y., (1976). Estimation of incident and reflected waves in random wave experiments. Proc. 15th Int. Coastal Engineering Conference (ASCE), 828-845.

Isaacson, M., (1991). Measurement of regular wave reflection. J. Waterway, Port, Coastal and Ocean Eng., 117, pp. 553-569.

Jarlan, G.E.,1961. A perforated vertical wall breakwater. Dock Harbour Auth. XII, 486: 394-398.

Kondo, H. (1979). Analysis of breakwaters having two porous walls. Proc. Coastal Structures '79, 2 , pp. 962-977.

Li, Y.C., Dong, G.H., Liu, H.J., Sun, D.P., 2003. The reflection of oblique incident waves by breakwaters with double-layered perforated wall. Coast. Eng. 50, 47-60.

Liu Y., Li, Y.C., Teng B. (2007) The reflection of oblique waves by an infinite number of partially perforated caissons. Ocean Eng. 34 (2007) 1965-1976.

Madsen, P.A. (1983) Wave reflection from a vertical permeable wave absorber. Coast. Eng. (7) pp. 381-396

Mansard, E. P. D., Funke, E. R., (1980). The measurement of incident and reflected spectra using a least square method. Proc. $17^{\text {th }}$ Int. Coastal Engineering Conference, (ASCE), 1, pp. 154-172.

Marks, M., Jarlan, G.E., 1968. Experimental study on a fixed perforated breakwater. Proc. $11^{\text {th }}$ Coastal Engineering Conf., London, vol. 2, pp. 1121-1140.

Postma, G.M., 1989. Wave reflection from rock slopes under random wave attack. MSc. Thesis, Delft University of Technology.

Suh, K.D., Choi, J.C., Kim, B.H., Park, W.S., Lee, K.S., 2001. Reflection of irregular waves from perforated-wall caisson breakwaters. Coastal Engineering, 44: 141-151.

Suh, K.D., Park, W.S. 1995. Wave reflection from perforated wall caisson breakwaters. Coast. Eng. 26, pp. 177-193.

Suh, K.D., Park, J.K., and Park, W.S. 2006. Wave reflection from partially perforated-wall caisson breakwater, Ocean Engineering, 33, 264-280.

Sunamura and Okazaki, 1996. Breaker types and wave reflection coefficient: laboratory relationship. $J$. Coastal Res., 12(1): 240-245.

Takahashi, A., Yasuo, K., Fujiwara, R., Isobe, M., 2002. Performance evaluation of perforated-wall caissons by VOF numerical simulations. Proc. 28th Int. Coastal Engineering Conference (ASCE), 1364-1376.

Thorton, E. B., Calhoun, R. J., (1972). Spectral resolution of breakwater reflected waves”. Proc. ASCE 98 (WW4), pp. 443-460.

Zhu, S., Chwang, A.T. (2002), Experimental studies on caisson-type porous seawalls. Experiments in Fluids 33 512-515 